\title{
El LADO NOCTURNO DE LA VIDA COTIDIANA: UN ANÁLISIS FEMINISTA DE LA PLANIFICACIÓN URBANA NOCTURNA
}

\author{
The Night Side of the Everyday Life: A Feminist Analysis \\ of Planning the Night
}

\author{
Sara Ortiz Escalante \\ saraortiz78@gmail.com \\ University of British Columbia
}

RESUMEN: Partiendo de una revisión bibliográfica realizada en el ámbito de la planificación urbana nocturna, este artículo plantea incluir una perspectiva de género interseccional en la planificación de las ciudades de noche. El artículo analiza estudios existentes sobre cómo los cuerpos de las mujeres han sido conceptualizados en el ámbito de la planificación urbana y en lo nocturno, cómo la planificación urbana ha abordado la esfera nocturna, y cómo el miedo y la seguridad afectan a la movilidad de las mujeres en la noche. La revisión bibliográfica revela que el rol de la planificación en relación a la noche ha sido regular y controlar lo que pasa en la noche y quién tiene derecho a la ciudad nocturna, pero la planificación urbana nocturna no ha incorporado la perspectiva de género interseccional, factor que dificulta la vida cotidiana diurna y nocturna de las personas que, debido al trabajo productivo, reproductivo o de cuidado, usan la ciudad en la noche de manera regular. El artículo propone incluir la diversidad de cuerpos sexuados en la planificación urbana nocturna, expandiendo el debate más allá de la economía nocturna de consumo y ocio, y visibilizando el rol que juegan las mujeres en la planificación de la noche.

Palabras Clave: género, planificación urbana, noche, seguridad, movilidad. 
ABSTRACT: Based on a literature review of research on nocturnal urban planning, this paper advocates including in the new urban agenda an intersectional gender perspective in planning the urban night. The paper analyzes the existing research on how gendered bodies have been conceptualized in planning and in the nocturnal context, how urban planning has approached the nocturnal sphere, and how fear and safety affect women's mobility at night. The literature review reveals the role of planning in relation to the nocturnal sphere as one of regulating and controlling what happens at night and who has the right to the night city. However, there is no gender and intersectional perspective in nocturnal planning, which encumbers the everyday/everynight life of those who, because of their productive, care and reproductive work, regularly use the city after dark. The paper concludes by advocating the inclusion of diverse gendered bodies in the new urban agenda and in planning the night, expanding this debate beyond the night-time economy of leisure and consumption, and making women's role visible in nocturnal planning.

KEYWORDS: gender, planning, night, safety, mobility.

RESUM: Partint d'una revisió bibliogràfica realitzada en l'àmbit de la planificació urbana nocturna, aquest article planteja incloure una perspectiva de gènere interseccional en la planificació de les ciutats de nit. L'article analitza estudis existents sobre com els cossos de les dones han estat conceptualitzats en l'àmbit de la planificació urbana i en el nocturn, com la planificació urbana ha abordat l'esfera nocturna, i com la por i la seguretat afecten la mobilitat de les dones a la nit. La revisió bibliogràfica revela que el paper de la planificació en relació a la nit ha estat regular i controlar allò que passa en la nit i qui té dret a la ciutat nocturna, però la planificació urbana nocturna no ha incorporat la perspectiva de gènere interseccional, factor que dificulta la vida quotidiana diürna i nocturna de les persones que, a causa del treball productiu, reproductiu o de cura, usen la ciutat en la nit de manera regular. L'article proposa incloure la diversitat de cossos sexuats en la planificació urbana nocturna, expandint el debat més enllà de l'economia nocturna de consum i oci, i visibilitzant el rol que juguen les dones en la planificació de la nit.

Paraules ClaU: gènere, planificació urbana, nit, seguretat, mobilitat. 


\section{Introducción}

$\mathrm{L}$ a historia de la planificación urbana ha omitido las contribuciones de las mujeres, las personas de orígenes étnicos diversos, la población indígena, las personas LGBTQ y otros grupos históricamente excluidos de las estructuras de poder y toma de decisiones (Sandercock, 1998; Fainstein and Servon, 2005). Bajo la premisa de planificar para el interés público, a menudo entendido como único, universal y estandarizado, por más de un siglo el urbanismo ha ignorado la diversidad de realidades en nuestra sociedad, y reproducido desigualdades, privilegiando a la gente más poderosa (Healey, 1997; Fainstein and Servon, 2005). En concreto, las mujeres han sido excluidas de la noche debido a cómo sus cuerpos han sido definidos y controlados socialmente. La noche ha sido históricamente conceptualizada como un tiempo y espacio peligroso y prohibido para las mujeres (Wilson, 1991; Hooper, 1998). En consecuencia, las mujeres que transgreden este imaginario y utilizan el espacio público en la noche son aún vistas como fuera de lugar en muchos contextos.

Desde la década de los 70 las urbanistas y geógrafas feministas han aportado una amplia variedad de reflexiones y contribuciones a la teoría y la práctica de la planificación urbana (e. g. Falú, 2009; Greed, 1994; Hayden, 1980; Leavitt, 2003; Muxí Martínez et al., 2011; Rahder y Altilia, 2004; Sandercock y Forsyth, 1992; Sandercock, 1998; Wilson 1991) que pueden ser aplicadas a la planificación urbana nocturna y que contribuyen al derecho de las mujeres a la ciudad.

Este artículo forma parte de una tesis doctoral ${ }^{1}$ que analiza cómo la planificación urbana aborda y responde a la vida cotidiana diurna y nocturna (Smith,

1. El título de la tesis es Mujeres transgresoras de espacio y tiempo: la planificación de la vida cotidiana diurna y nocturna de las mujeres que trabajan de noche. Esta investigación estudia cómo la planificación urbana aborda la vida cotidiana diurna y nocturna de las mujeres que trabajan de noche en el Área Metropolitana de Barcelona. Se utiliza la metodología de investigación-acción participativa feminista para explorar cómo las mujeres que trabajan de noche pueden influenciar las políticas públicas y las prácticas urbanas. La investigación se desarrolla en colaboración con Fundació Àmbit Prevenció, las mujeres que trabajan de noche en el Hospital de Bellvitge, la Secretaria de la Mujer de CCOO en Barcelona y el Baix Llobregat, Ca la Dona, e Irídia. 24 mujeres que trabajan de noche en la zona metropolitana de Barcelona son co-investigadoras en este proyecto. Esta investigación recibe el apoyo de University of British Columbia Public Scholar Initiative, y el Ajuntament de Barcelona. 
1990) de las mujeres que trabajan de noche, y en particular examinando dos aspectos interrelacionados de la planificación urbana que afecta a la vida cotidiana nocturna de las mujeres: el miedo/la seguridad y la movilidad. Esta investigación explora desde un análisis feminista interseccional, ${ }^{2}$ cómo el miedo y la percepción de seguridad están vinculados a la construcción sociocultural de los cuerpos sexuados en los espacios públicos y cómo éstos influyen en la movilidad de las mujeres trabajadoras y el derecho a la ciudad de noche. La investigación se encuentra en la actualidad en fase de análisis de datos cualitativos recogidos conjuntamente con las mujeres en el Área Metropolitana de Barcelona. Este artículo se centra en el marco teórico con el fin de visibilizar la necesidad de incluir una perspectiva de género cuando se planifica la ciudad de noche.

El objetivo de este artículo es visibilizar que la planificación urbana nocturna se ha centrado sobre todo en regular y controlar lo que pasa en la noche y quién tiene derecho a la ciudad nocturna. Pero se ha hecho muy poco en facilitar la vida cotidiana diurna y nocturna de las personas que, debido al trabajo productivo, reproductivo y de cuidado, utilizan la ciudad de noche de forma regular. Además, el artículo revela que la planificación urbana nocturna no incluye una perspectiva de género interseccional, sino que ha incluido los cuerpos sexuados de hombres blancos jóvenes, pero continúa excluyendo otros cuerpos sexuados: los cuerpos de las mujeres, especialmente de mujeres de bajos ingresos, mujeres migrantes, mujeres de diferentes orígenes, así como la población transgénero y los hombres que no son blancos.

Este artículo aboga por la necesidad de incluir el estudio de la vida cotidiana diurna y nocturna en la planificación urbana con el objetivo de: visibilizar cómo las realidades diversas de género no se han incluido en las políticas de planificación urbana nocturna; dar la misma relevancia a las contribuciones de las mujeres en el trabajo remunerado formal e informal que en el trabajo reproductivo, doméstico, de cuidado y el trabajo comunitario; y enfatizar cómo el trabajo de las mujeres en la noche es esencial para que

2. El término feminismo interseccional rompe con concepciones esencialistas de ser una «mujer» y analiza cómo diferentes fuentes estructurales de desigualdad como el género, la raza, la etnia, la clase, la identidad sexual, el origen y el estatus migratorio se construyen socialmente y están interrelacionadas (Crenshaw, 1991). 
el mundo siga funcionando de día. Finalmente, incluir una perspectiva de género al planificar la vida urbana nocturna es necesario para presionar a las políticas públicas para que respondan a las necesidades resultantes de la doble presencia de las mujeres en la economía nocturna remunerada y el trabajo doméstico y de cuidado no remunerado. El urbanismo puede contribuir a transformar el trabajo no remunerado en una responsabilidad social y colectiva en vez de ser una carga que a menudo recae en las mujeres.

A continuación se hace una revisión bibliográfica de la literatura que analiza la interrelación entre el género, el urbanismo y la noche. El artículo concluye con algunas reflexiones para incluir una perspectiva de género en la planificación urbana nocturna.

\section{Una visión de género del urbanismo y la noche}

Analizar cómo la perspectiva de género se ha incluido/excluido en la planificación urbana nocturna implica examinar la investigación desarrollada acerca de cómo el género ha sido conceptualizado por el urbanismo y en la noche, cómo la planificación urbana ha trabajado la esfera nocturna, y cómo el miedo y la percepción de seguridad afectan a la movilidad nocturna de las mujeres.

\subsection{El género en la planificación urbana nocturna}

Investigadoras feministas han documentado cómo la diversidad sexual, racial y de género de los cuerpos ha sido construida y regulada a través del urbanismo (Doan, 2010; Green \& Singleton, 2006; Hooper, 1998; Sandercock, 1999). Los cuerpos de las mujeres en particular han sido conceptualizados en el espacio público como una amenaza al orden social, como una fuente de miedo que «deshace la idea del plan» (Sandercock, 2003, p. 30); pero también como un cuerpo vulnerable y objetivado para ser dominado (Wesely \& Gaarder, 2004). Esta exclusión se ha reforzado a través de las connotaciones históricas asociadas a las mujeres en los espacios públicos, y a la falsa dicotomía público/privado. Por ejemplo, el término mujer pública ha sido asociado con la prostitución, ser una mujer «no respetable», ser una 
tentación sexual para la auto-disciplina masculina, para el hombre público, que es entendido como el «hombre de estado» (Wilson, 1991; Duncan, 1996; Hooper, 1998; Massolo, 2007). En consecuencia, el urbanismo ha sido cómplice en la reproducción de la dicotomía opresora público/privado, que posiciona a las mujeres en la esfera privada asociada con lo doméstico, lo emocional, lo corporal, la familia y el trabajo no remunerado y/o informal; y a los hombres en la esfera pública de la producción, del trabajo remunerado, lo racional, lo a-corporal, el mercado, el estado y el poder (Duncan, 1996; Sweet \& Ortiz Escalante, 2010).

Además de ser androcéntrica, la dicotomía público/privado es etnocentrista y opresiva contra las personas queer y transgénero. Es etnocentrista porque esta dicotomía es aún más limitante cuando se utiliza en contextos de asentamientos informales donde la casa no existe porque las personas viven en chabolas, es decir, en estructuras muy vulnerables en las que las puertas o ventanas no siempre se pueden cerrar de manera segura (Meth, 2003). Es opresiva contra las personas queer y transgénero porque la división sexual del espacio fuerza a las personas a responder a expectativas hegemónicas de comportamientos de género que responden al binomio masculino/femenino (Doan, 2010).

La reproducción de este binomio y la exclusión de las mujeres de la esfera pública resulta más evidente cuando oscurece. Las mujeres han sido excluidas de la noche por cómo sus cuerpos han sido definidos y controlados. El contexto y las identidades interseccionales de las mujeres limita su actividad nocturna. La noche ha sido históricamente conceptualizada como un espacio-tiempo prohibido y peligroso para las mujeres. La expresión «mujer de la noche», al igual que «mujer pública», está cargada negativamente y también asociada con la prostitución, el desorden o el ser una "perdida» (Patel, 2010). En consecuencia, las mujeres que trasgreden este imaginario y utilizan el espacio público en la noche son vistas como fuera de lugar en muchos contextos sociales.

Incluir una perspectiva de género interseccional en la agenda urbana puede visibilizar una relación más fluida entre los cuerpos sexuados y la ciudad (Milroy in Miranne and Young, 2000; Doan, 2010; Sweet and Ortiz Escalante, 2014), entendiendo los cuerpos como la escala espacial que conecta los 
espacios públicos y privados, como un espacio biográfico, como un espacio de memoria de las violencias, pero también como un espacio de resistencia (Falú, 2009; Vargas, 2009). Tomar el cuerpo como un espacio de auto-conciencia y resistencia, como un espacio único y privado, para poder apropiarlo primero como mujeres, para así poder apropiarse luego de otros territorios: la casa, el barrio, la ciudad, el país (Falú, 2009; Vargas, 2009).

Pero también una perspectiva de género interseccional en la agenda urbana implica incorporar la vida cotidiana diurna y nocturna de las mujeres como una fuente de conocimiento y una metodología. Analizar la vida cotidiana significa dar el mismo valor a las necesidades provenientes de la esfera productiva del trabajo remunerado que a las esferas no remuneradas del trabajo doméstico, reproductivo y de cuidado y del trabajo comunitario, con el fin de promover una división del trabajo más equitativa, además de visibilizar las contribuciones de las mujeres en la economía doméstica y comunitaria (Healey, 1997; Gilroy and Booth, 1999; Bofill Levi, 2005; Muxí Martínez et al., 2011). Hay pocas referencias a la vida cotidiana nocturna en la literatura feminista sobre vida cotidiana. La única referencia específica proviene de la socióloga Dorothy Smith (1990) quien habla sobre la vida cotidiana diurna y nocturna (everyday/ everynight life) y de quién tomó prestado el término vida cotidiana nocturna (everynight), para argumentar que el concepto de vida cotidiana necesita ser ampliado e incluir tanto el día como la noche.

\subsection{Planificando la noche}

La noche es un término controvertido que ha sido social y culturalmente moldeado a través de la historia. Cómo se conceptualiza la noche y cuándo empieza y termina también varia entre culturas, periodos históricos y localización geográfica. La expansión del alumbrado público en el sigo XIX cambió el significado y uso de la ciudad en la noche, y facilitó el aumento de la vida nocturna sobre todo en las áreas urbanas, expandiendo la economía nocturna (Melbin, 1978, 1987; Schivelbusch, 1988; Edensor, 2013).

En las sociedades occidentales, la noche ha sido asociada con el miedo, el caos, el demonio, el pecado, la muerte y el lado oscuro de la sociedad (Schivelbusch, 1988; Palmer, 2000; Edensor, 2013); y el día con la creación del mundo, Dios, lo «bueno», lo «seguro». Este imaginario occidental euro- 
céntrico ha asociado la noche con aquellas personas que transgreden el orden racional de la sociedad, con las prácticas, ocupaciones, ideas y sexualidades transgresoras, por ejemplo, la prostitución, los y las revolucionarias, la escena musical, o el tráfico de drogas (Palmer, 2000). Sin embargo, en culturas no-occidentales, hay también percepciones positivas de la noche, donde las personas utilizan este tiempo para rituales comunitarios, eventos familiares o actividades religiosas (Amid, 2013).

Los relatos históricos sobre la noche también han reforzado los dualismos entre el día y la noche, lo bueno y lo malo, incluso lo masculino y lo femenino (Melbin, 1987; Schivelbusch, 1988; Palmer, 2000). Este dualismo simplista entre el día y la noche, ha construido estos términos como opuestos, obviando la diversidad de cada condición y cómo el alumbrado público ha complicado este dualismo, al mismo tiempo que ha legitimado agendas políticas y sociales conservadoras que limitan el acceso a la noche de ciertos grupos de personas (Gallan \& Gibson, 2011).

En el ámbito de la planificación urbana, la mayor parte de los estudios sobre la noche se centran en lo que se conoce como «la economía nocturna». Son estudios mayoritariamente desarrollados en el contexto occidental, sobre todo en el Reino Unido, y en menos grado en los Estados Unidos y Australia. Estos estudios se han enfocado en la «economía nocturna» de los centros de ciudades que buscan la revitalización económica, dando énfasis a las actividades de ocio y entretenimiento, generalmente asociadas con el consumo de alcohol. El término «economía nocturna» fue usado por primera vez en los 90 por Franco Bianchini de la organización de ciudades creativas Comedia Consultancy (Bianchini, 1995; van Liempt et al., 2014; Shaw, 2014). La economía nocturna se refería inicialmente a la multi-industria de la producción cultural nocturna, en la que el alcohol y el ocio eran parten de la actividad nocturna (Shaw, 2014). Sin embargo, la mayoría de políticas relacionadas con la economía nocturna se han centrado en la desregularización del consumo de ocio y alcohol (Shaw, 2014; van Liempt et al., 2014), y se han convertido en estrategias neoliberales para «ciudades reinventándose a sí mismas como lugares de consumo» (van Liempt et al., 2014, p. 6). En resumen, la mayoría de los estudios sobre la economía nocturna analizan el lado consumista de la ciudad 24-horas en zonas céntricas, 
y los problemas que se derivan como la violencia y la inseguridad (Bromley et al., 2000; Thomas \& Bromley, 2000; Bromley et al., 2003; Crawford \& Flint, 2009; Beer, 2011; Evans, 2012; Eldridge \& Roberts, 2013).

Algunos estudios han incluido una perspectiva de género o un análisis crítico de temas de etnia y raza (Roberts, 2006; Talbot, 2007; Sheard, 2011; Waitt \& Gorman-Murray, 2011; Roberts and Eldridge, 2012; Schwanen et al., 2012; Roberts, 2013). Los análisis de género miran la exclusión, la desigualdad o el acceso a la economía nocturna. Estos estudios revelan que las formas dominantes de vida nocturna están protagonizadas por sujetos masculinos y heterosexuales (Chatterton \& Hollands, 2003; Sheard, 2011; Hubbard \& Colosi, 2013). Por ejemplo, el acceso de las mujeres jóvenes a la economía nocturna se ha hecho en términos masculinos, adoptando patrones masculinos de beber y emborracharse y de involucrarse en violencia (Chatterton \& Hollands, 2003; Waitt \& Gorman-Murray, 2011), o evaluando el riesgo de las mujeres de ser abusadas sexualmente (Sheard, 2011).

Pocos estudios miran el lado productivo de la economía nocturna (e. g. Shaw, 2014), a pesar de que la noche ha sido siempre un tiempo de producción, y las mujeres han formado siempre parte de la noche como trabajadoras a pesar de intentos de excluirlas a lo largo de la historia (Melbin, 1987; Patel, 2010; Lowson \& Arber, 2013). Estudios sobre las experiencias de género del trabajo nocturno se encuentran sobre todo en otras áreas de estudio como la sociología o las relaciones laborales, y se enfocan más en los aspectos fisiológicos que en las consecuencias sociales de ser trabajadoras nocturnas. Los poco ejemplos de estudios que contemplan aspectos sociales son mayoritariamente cuantitativos (Lowson \& Arber, 2013). Los estudios sociales sobre la noche que incluyen una perspectiva de género examinan el impacto del trabajo nocturno de las mujeres en las relaciones familiares (Melbin, 1987; Garey, 1995; Lowson \& Arber, 2013).

En general, la investigación sobre planificación urbana nocturna ignora los ciclos nocturnos fuera de los centros urbanos, sin analizar otras partes de la ciudad $\mathrm{u}$ otro tipo de actividades nocturnas. Existen pocos estudios sobre personas que utilizan la noche para actividades que no son de ocio o en contextos fuera del mundo occidental (Patel, 2010; Amid, 2013). 
Por lo tanto, la planificación urbana nocturna se ha enfocado en una parte pequeña de la vida nocturna: el lado consumista de la economía nocturna que está relacionado con el consumo de ocio y alcohol en centros urbanos de ciudades occidentales. En general, se ha romantizado a las personas usuarias de la noche como un grupo especial de población, sin reconocer que la noche también es un espacio de trabajo, cuidado y reproducción, un espacio de la vida cotidiana diurna y nocturna, que no tiene ningún glamur para aquellas personas que se ven obligadas a trabajar en el turno de noche.

En resumen, existe la necesidad de incluir en la planificación urbana nocturna, políticas públicas del lado productivo de la economía nocturna y desde una perspectiva de género interseccional, que vayan más allá de los centros urbanos a otros barrios, centros de trabajos, pueblos y hogares; que rompan con la cultura nocturna masculina; que visibilicen las necesidades de la vida cotidiana diurna y nocturna y analicen cómo el urbanismo puede contribuir a mejorar la calidad de vida y el derecho a la ciudad.

\subsection{Miedo, percepción de seguridad y movilidad}

Los estudios de urbanismo feminista hacen referencia a la noche en relación al tema del miedo, la seguridad y la movilidad. Estos estudios analizan cómo las percepciones de miedo de las mujeres incrementan en la noche (Valentine, 1989; Koskela, 1999; Pain, 2001; Loukaitou-Sideris, 2006; Dammert, 2007; Falú, 2009) o examinan cómo el miedo y la seguridad limitan la movilidad de las mujeres (Atkins, 1989; Ganjavi et al., 2000; Carter, 2005; Whitzman, 2012).

El miedo y la seguridad han sido estudiados profundamente desde el urbanismo. «La planificación urbana y los discursos de gestión urbana están, y siempre han estado, saturados con miedo. La historia de la planificación podría reescribirse como el intento de gestionar el miedo en la ciudad» (Sandercock, 2002, p. 203). Al mismo tiempo, los estudios han demostrado cómo el miedo y la seguridad limitan la movilidad de las mujeres, sobre todo en la noche (Pain, 1991, 1997; Koskela, 1999; Loukaitou-Sideris, 2005).

Desde el urbanismo, muchas teorías e intervenciones se han centrado en cómo controlar y prevenir el crimen a través del diseño del entorno físico, por ejemplo, la Prevención del Crimen a través del Diseño Ambiental (Crime 
Prevention Through Environmental Design - CPTED) o los programas de Ciudades Seguras. Sin embargo, estas iniciativas responden mayoritariamente a crímenes perpetrados por personas extrañas en el espacio público. Las feministas han criticado estas iniciativas por ser ciegas al género, enfocándose sólo en el aspecto físico de diseñar cómo eliminar el miedo y no incluyendo un análisis social de cómo la seguridad se percibe de manera diferente dependiendo del sexo, el género y otras variables que se entrecruzan (Koskela \& Pain, 2000; Pain, 2001; Sweet \& Ortiz Escalante, 2010).

Los estudios de urbanismo feminista se centran en el miedo y la percepción de seguridad y no en el crimen, porque el crimen sólo se refiere a aquellos actos violentos reconocidos legalmente, los cuales varían dependiendo del contexto. Sin embargo, el miedo y la percepción de seguridad son conceptos que toman una aproximación más compleja al impacto de la violencia en las vidas de las personas, no sólo en el espacio público y perpetrada por personas extrañas, sino como un continuo entre la esfera pública y privada. De hecho, examinar el miedo y la seguridad permite incluir el acoso sexual callejero, «una forma no-criminal de violencia callejera que tiene un impacto muy marcado en el acceso de las mujeres al espacio urbano» (Koskela \& Tani, 2000).

El miedo se puede definir como la respuesta corporal, emocional y práctica de las personas y comunidades hacia la violencia (Pain, 2001; Koskela, 2010). El miedo está basado en relaciones de poder de género en los espacios (Epstein, 1998; Koskela, 1999, 2010; Dammert, 2007) y se reproduce en las prácticas de la vida cotidiana (Gordon \& Riger, 1989; Valentine, 1989; Koskela, 2010; Sandberg \& Rönnblom, 2014). Estudios desde diferentes ámbitos como la sociología, la psicología evolutiva o la pedagogía documentan cómo el miedo se reproduce a través del proceso de socialización mediante la reproducción de los roles de género tradicionales que definen a las mujeres como vulnerables y a los hombres como fuertes y agresivos. Esta producción social del miedo se transmite a través de canales formales e informales, desde mensajes de alerta recibidos en la unidad familiar, hasta las noticias, las conversaciones diarias o los consejos de prevención provenientes de organismos policiales (Mackie, 1987; Maccoby, 1992; Valentine, 1992; Stockard, 1999; Dammert, 2007; Koskela, 2010). 
También existen diferencias de género a la hora de denunciar la violencia y el miedo (Pain, 1997; Dammert, 2007; Koskela, 2010). La gran mayoría de la violencia perpetrada contra las mujeres pasa en la esfera privada y por parte de gente conocida (Stanko, 1988; Valentine, 1989, 1992; Pain, 1997; Sweet \& Ortiz Escalante, 2010). La naturaleza íntima y sexual de esta violencia contribuye a que no se denuncie, porque las mujeres tienen miedo de represalias o porque la violencia está relacionada con sus cuerpos sexuados (Koskela, 2010; Falú, 2011; Sweet \& Ortiz Escalante, 2014). Por otro lado, las experiencias y las manifestaciones del miedo también tienen género y se comunican también de forma diferente. Las mujeres tienden a tener miedo a la violencia sexual y la violación, el tipo de violencia que ataca nuestro cuerpo e intimidad (Pain, 1991; Falú, 2011; Sweet \& Ortiz Escalante, 2014). Además, las mujeres tienen más tendencia a adaptar y restringir su vida cotidiana debido a la violencia (Pain, 1991).

Tal y como se ha mencionado anteriormente, el miedo y la seguridad afectan a la vida cotidiana de las mujeres y su movilidad, uso y participación en la ciudad (Pain, 1991; Moser, 2012), y estas limitaciones se vuelven más severas en la noche (Lynch \& Atkins, 1988; Atkins, 1989; Pain, 1991, 1997; Koskela, 1999; Ganjavi et al., 2000; Carter, 2005; Loukaitou-Sideris, 2005; Laub, 2007; Morey, 2007; Whitzman, 2012). Las investigadoras feministas han adoptado el término movilidad con el fin de romper las barreras del urbanismo tradicional y los estudios de transporte, y poder examinar al completo la complejidad de actividades interrelacionadas que incluyen la casa, la comunidad y la sociedad, en vez de ver el transporte como una opción individual y unidireccional de casa al trabajo que prioriza los desplazamientos relacionados con el trabajo remunerado (Law, 1999; Hanson, 2010; Miralles-Guasch, 2010). Los estudios sobre patrones de movilidad de las mujeres en los contextos europeos y norteamericanos han demostrado que las mujeres tienen unos patrones de movilidad más sostenibles, complejos y diversos que los hombres durante el día (Grieco et al., 1989, Grieco \& McQuaid, 2012; Hanson \& Hanson, 1980, 1981, 1985; Law, 1999; Hanson, 2010; Miralles-Guasch \& Martínez-Melo, 2012; Miralles-Guasch, 2010). Sin embargo, la movilidad de las mujeres se puede ver paralizada en la noche debido al miedo. En la noche las mujeres evitan ciertas partes de la ciudad, no usan ciertos modos de transporte, o dejan de salir a la calle (Atkins, 1989; Ganjavi 
et al., 2000; Carter, 2005; Loukaitou-Sideris, 2005, 2006; Whitzman, 2012). Es imprescindible que el urbanismo responda a esta paradoja con el fin de garantizar al completo el derecho de las mujeres a la ciudad tanto de día como de noche.

\section{Hacia una planificación urbana nocturna y feminista}

Esta revisión confirma que el enfoque prioritario de la planificación urbana nocturna ha sido regular y controlar lo que pasa en la noche y quién tiene derecho a la noche. Pero poco ha hecho para facilitar la vida cotidiana diurna y nocturna de la gente que utiliza la noche de manera regular. La vida nocturna parece ser percibida como una excepción, incluso para el ocio, a pesar que salir de noche es una rutina de fin de semana de ciertos grupos de personas. Además, el urbanismo ha apoyado el modelo neoliberal de maximizar los beneficios de la economía nocturna y abordar los problemas que pueden interferir en este negocio, como el botellón, la violencia vinculada al consumo de alcohol o las quejas vecinales. En este sentido, la planificación urbana nocturna, responde a políticas neoliberales e ignora las necesidades de la vida cotidiana diurna y nocturna. Es decir, en general, ha ignorado la vida cotidiana nocturna de aquellas personas que debido al trabajo productivo, reproductivo y de cuidado utilizan la ciudad de noche de forma regular. Al mismo tiempo, la planificación urbana nocturna carece de una perspectiva de género interseccional. Ha incluido los cuerpos de hombres jóvenes adultos blancos, pero continúa excluyendo otros cuerpos por su género: los cuerpos de mujeres, en concreto, mujeres de bajos recursos, mujeres de orígenes diversos, mujeres migrantes, así como población transgénero y hombres no-blancos.

Es imprescindible expandir el debate de la planificación urbana nocturna más allá de la economía nocturna del ocio y el consumo y visibilizar las contribuciones de las mujeres a las diferentes economías nocturnas y en otras partes de las zonas urbanas más allá del centro. Incorporar las contribuciones que el feminismo ha hecho, como el análisis de los cuerpos como una escala espacial, analizando cómo los cuerpos sexuados sienten, perciben, viven y resisten la noche urbana permitirá un mejor análisis del rol que el miedo y la seguridad juegan en la vida cotidiana diurna y nocturna de las mujeres, así como el uso de la vida cotidiana diurna y nocturna como fuente de conoci- 
miento y metodología puede visibilizar las experiencias de las mujeres que trabajan de noche.

Las políticas de planificación urbana nocturna deben incluir los cuerpos como escala espacial que desenfatice la división público-privada y entienda cómo las mujeres viven y sienten las percepciones de miedo, así como incluir a las mujeres como sujetos de cambio y transformación que deben estar presentes en todas las fases de la planificación desde el diagnóstico hasta la evaluación. El uso de los cuerpos como espacio puede también enfatizar el continuo entre los espacios públicos y privados, y remarcar las experiencias corporales de género en la casa, la comunidad, el barrio y la ciudad. También, el cuestionamiento y la deconstrucción feminista de la dicotomía público/ privado se puede aplicar a la dicotomía día/noche, que también construye y perpetúa concepciones patriarcales del lugar de las mujeres en la sociedad. En relación a esto, agregar lo nocturno al estudio de la vida cotidiana puede visibilizar las actividades rutinarias de la noche, y cuestionar el imaginario de que la noche es un tiempo-espacio de excepción y transgresión. También, las políticas de planificación urbana nocturna deben responder a cómo el miedo, la seguridad y el riesgo afectan a las mujeres que trabajan de noche, incluyendo un análisis interseccional que rompa con argumentos esencialistas sobre el miedo de hombres y mujeres. Finalmente, la planificación urbana nocturna, tiene la obligación de responder a la paradoja de movilidad de las mujeres. Es inconcebible que en términos generales, las mujeres tengan una movilidad más sostenible, compleja y diversa que los hombres durante el día, pero que se pueda ver paralizada en la noche por el miedo a la violencia. Existen multitud de estudios que muestran esta paradoja y las políticas de planificación urbana no han puesto la atención que se merece en este problema.

La vida cotidiana diurna y nocturna de las mujeres debe incorporarse en las políticas de planificación urbana nocturna como una fuente de conocimiento y una metodología. Las experiencias de las mujeres deben ser escuchadas e incluidas en el urbanismo para aprender cómo la vida cotidiana diurna y nocturna funciona en todas sus esferas (productiva, reproductiva, comunitaria y personal). La inclusión de la vida cotidiana diurna y nocturna de las mujeres ayudará a planificar mejor las ciudades, con el objetivo de que el trabajo doméstico y de cuidado se convierta en una responsabilidad social 
y colectiva, algo a lo que tenemos que responder como sociedad, como comunidad, como gobierno municipal, como región y como país, y no algo que una persona, familia o unidad de convivencia tiene que gestionar sin apoyo externo. Esto contribuiría a valorar las tareas domésticas y de cuidado y eliminar el peso de la responsabilidad que hasta ahora recae mayoritariamente sobre las mujeres.

Analizando las rutinas de las mujeres de día y de noche, el tipo de actividades que desarrollan, con quién las desarrollan, a qué hora y en qué modo de transporte ayudaría a entender: el rol de la movilidad en el acceso a la vida nocturna; las contribuciones de las trabajadoras de noche a la economía nocturna remunerada y no remunerada; la negociación de la movilidad en la esfera privada; el rol del transporte público; así como problemas de movilidad forzada o inmovilidad. En resumen, analizar la vida de las mujeres en la noche puede visibilizar el uso y la apropiación de las mujeres del territorio en la noche, y reclamar que la noche también es nuestra.

\section{Referencias}

Amid, A. (2013). «Night, Space and Urban design: Case study of Mashhad, Iran. PhD dissertation». School of Architecture and the Built Environment, University of Westminster.

Atkins, S. (1989). «Women, Travel and Personal Security». In M. Grieco, L. Pickup \& R. Whipp: Gender, transport, and employment: the impact of travel constraints. Gower Publishing Company.

BeER, C. (2011). «Centres that Never Sleep? Planning for the Night-Time Economy Within the Commercial Centres of Australian Cities». Australian Planner, 48(3), pp. 141-147.

Bofill LeVI, A. (2005). «Planejament urbanístic, espais urbans i espais interiors des de la perspectiva de les dones». Quaderns de l'Institut núm. 6, Institut Català de les Dones, Departament de Política Territorial i Obres Públiques, Generalitat de Catalunya, Barcelona. Recuperado de http://dones.gencat.cat/web/sites/dones/.content/03_serveis/docs/ publicacions_quaderns06.pdf 
Bromley, R. D., Tallon, A. R., \& Thomas, C. J. (2003). «Disaggregating the Space-time Layers of City-centre Activities and their Users». Environment and Planning A, 35(10), pp. 1831-1852.

Bromley, R., Thomas, C., \& Millie, A. (2000). «Exploring Safety Concerns in the Night-time City: Revitalising the Evening Economy». Town Planning Review, 71(1), 71.

Carter, M. (2005). «Gender Differences in Experience With and Fear of Crime in Relation to Public Transport». Research on women's issues in transportation, Vol. 2, Conference proceedings, 35, 100. Washington, DC: Transportation Research Board.

Crawford, A., \& Flint, J. (2009). Urban Safety, Anti-Social Behaviour and the Night-Time Economy. Criminology and Criminal Justice, 9(4), pp. 403-413.

Crenshaw, K. (1991). «Mapping the Margins: Intersectionality, Identity Politics, and Violence Against Women of Color». Stanford law review, pp. 1241-1299.

Dammert, L. (2007). Entre el temor difuso y la realidad de la victimización femenina en América Latina. En A. Falú, O. Segovia \& M. Alonso, Ciudades para convivir: sin violencia hacia las mujeres, debates para la construcción de propuestas. Santiago de Chile: Ediciones Sur.

Doan, P. L. (2010). «The Tyranny of Gendered Spaces-Reflections from Beyond the Gender Dichotomy». Gender, Place and Culture, 17(5), pp. 635-654.

Duncan, N. (1996). Renegotiating Gender and Sexuality in Public and Private Spaces. En Duncan, N. (ed.), BodySpace. Destabilizing geographies of gender and sexuality. Routledge

Edensor, T. (2013). «The Gloomy City: Rethinking the Relationship between Light and Dark». Urban Studies, 0042098013504009. 
Eldridge, A., \& Roberts, M. (2013). «Re-populating the Nighttime City: Hospitality and Gender». En Space-Time Design of the Public City. Springer Netherlands.

EpsteIn, D. (1998). Afraid/Not. Psychoanalytic Directions for an Insurgent Planning Historiography. En L. Sandercock (Ed.), Making the invisible visible: A multicultural planning history. California: University of California Press

Evans, G. (2012). «Hold Back the Night: Nuit Blanche and All-night Events in Capital Cities». Current Issues in Tourism, 15(1-2), 35-49.

Fainstein, S. S., \& Servon, L. J. (EDs.) (2005). Gender and planning: A reader. Rutgers University Press.

FALÚ, A. (2009a). Mujeres en la Ciudad: De violencias y Derechos. Santiago de chile: Red Mujer y Hábitat de América Latina. Ediciones Sur

- (2011). «Restricciones ciudadanas: las violencias de género en el espacio público». En M. Largarde \& A. Valcárcel (coords.), Feminismo, género e igualdad. Ed. Pensamiento Iberoamericano.

Gallan, B. \& Gibson, C. R. (2011). «New Dawn or New Dusk? Beyond the Binary of Day and Night». Environment and Planning A, 43, 2509-2515.

GanjaVi, O., LeBrasseur, R., \& Whissell, R. (2000). «Night Walking Safety and Overall Satisfaction with Police Services». Policing: An International Journal of Police Strategies \& Management, 23(1), 22-37.

Gilroy, R., \& Bоотн, C. (1999). «Building an Infrastructure for Everyday Lives». European Planning Studies, 7(3), 307-324.

Gordon L. \& Riger S. (1989). The Female Fear. New York: Free Press.

Greed, C. (1994). Women and planning: creating gendered realities. Taylor $\&$ Francis.

Green, E., \& Singleton, C. (2006). «Risky Bodies at Leisure: Young Women Negotiating Space and Place». Sociology, 40(5), 853-871. 
Grieco, M., Pickup, L., \& Whipp, R. (1989). Gender, Transport and Employment. The Impact of Travel Constraints. Aldershot, uK: Gower Publishing Company.

Grieco, M., AND McQuaid R. (eds.) (2012). «Gender and Transport: An Editorial Introduction». Research in Transportation Economics 34, 1-2.

Hanson, S. (2010). «Gender and Mobility: New Approaches for Informing Sustainability». Gender, Place and Culture, 17, 1, 5-23.

- Hanson, P. (1980). "Gender and Urban Activity Pattern in Uppsala Sweden». American Geographical Society, 70:3, 291-299.

- Hanson, P. (1981). «The Travel-Activity Patterns of Urban Residents: Dimension and Relationships to Sociodemographic Charateristics». Economic Geography, 57, 4, 332-347.

- Johnston, I. (1985). «Gender Differences in Work, Tavel Lenght: Explanations and Implications». Urban Geography, 6, 3, 193-219.

Hayden, D. (1980). «What Would a Non-Sexist City Be Like? Speculations on Housing, Urban Design, and Human Work». Signs, 5(3), S170-S187.

Healey, P. (1997). Chapter 4: «Everyday Life and Local Environments». En Collaborative Planning. Shaping Places in fragmented societies. Macmillan Press.

Hooper, B. (1998). «The Poem of Male Desires». En Sandercock, L., Making the invisible visible: a multicultural planning history. California: University of California Press.

Koskela, H. (1999). «“Gendered Exclusions”: Women’s Fear of Violence and Changing Relations to Space». Geografiska Annaler: Series B, Human Geography, 81(2), 111-124.

— PaIn, R. (2000). «Revisiting Fear and Place: Women's Fear of Attack and the Built Environment». Geoforum, 31(2), 269-280. 
— TANI, S. (2005). «"Sold out!” Women’s Practices of Resistance Against Prostitution Related Sexual Harassment». Women's Studies International Forum, 28(5), 418-429.

KosKela. H. (2010). Fear and its Others. En S. Smith (ed.), The SAGE handbook of social geographies. SAGE Publications.

LAub, C. (2007). «Violencia urbana, violencia de género y políticas de seguridad ciudadana». En A. Falú, O. Segovia, \& M. Alonso, Ciudades para convivir: sin violencia hacia las mujeres, debates para la construcción de propuestas. Santiago de Chile: Ediciones Sur.

LAw, R. (1999). «Beyond "Women and Transport": Towards new Geographies of Gender and Daily Mobility». Progress in Human Geography, 23(4), 567-588.

LeavitT, J. (2003). «Where's the Gender in Community Development?». Signs, 29(1), 207-231.

Loukaitou-Sideris, A. (2005). «Is It Safe to Walk Here?». Research on women's issues in transportation, 102.

— (2006). «Is it Safe to Walk? Neighborhood Safety and Security Considerations and Their Effects on Walking». Journal of Planning Literature, 20(3), 219-232.

Lykogianni, R. (2008). «Tracing Multicultural Cities From the Perspective of Women $>$ S Everyday Lives». European Urban and Regional Studies, 15(2), 133-143.

Lynch, G., \& Atkins, S. (1988). «The Influence of Personal Security Fears on Women's Travel Patterns». Transportation 15, 257-77.

MAсCOBY, E. E. (1992). «The Role of Parents in the Socialization of Children: An Historical Overview». Developmental psychology, 28(6), 1006.

MaCkIE, M. (1987). Constructing Women and Men: Gender Socialization. Holt, Rinehart and Winston of Canada. 
Massolo, A. (2007). «Análisis y propuestas para la acción de los gobiernos locales en la seguridad de las mujeres en las ciudades». En A. Falú, O. Segovia, \& M. Alonso, Ciudades para convivir: sin violencia hacia las mujeres, debates para la construcción de propuestas. Santiago de Chile: Ediciones Sur.

Melbin, M. (1978). «Night as Frontier». American Sociological Review, 3-22.

- (1987). Night as Frontier: Colonizing the World After Dark. New York: Free Press.

Метн, P. (2003). «Rethinking the "Domus" in Domestic Violence: Homelessness, Space and Domestic Violence in South Africa». Geoforum, 34(3), 317-327.

Miralles Guasch, C. (2010). Dones, mobilitat, temps i ciutats. Col· lecció Quaderns de l'Institut 14, perspectives des del feminisme. Barcelona: Institut Català de les Dones.

Miralles Guasch, C., \& Martínez Melo, M. (2012). «Las divergencias de género en las pautas de movilidad en Cataluña, según edad y tamaño del municipio». Doi: 10.5212/Rlagg. v. 3. i2. 049060. Revista Latino-Americana de Geografia e Gênero, 3(2), 49-60.

Miranne, K. B., \& Young, A. H. (Eds.). (2000). Gendering the City: Women, Boundaries, and Visions of Urban Life. Rowman \& Littlefield.

Morey, P. (2007). «Introducción: Violencia de género: hacia una comprensión global». En Falú, A., Segovia, O., \& Alonso, M., Ciudades para convivir: sin violencia hacia las mujeres, debates para la construcción de propuestas. Santiago de Chile: Ediciones Sur.

Moser, C. (2012). «Mainstreaming Women>s Safety in Cities into Gender-based Policy and Programmes». Gender \& Development, 20(3), 435-452.

Muxí Martínez, Z., Casanovas, R., Ciocoletto, A., Fonseca, M., \& GutiérRez Valdivia, B. (2011). «¿Qué aporta la perspectiva de género al urbanismo?». Feminismos, 17, 105-129. 
PaIn, R. (1991). «Space, Sexual Violence and Social Control: Integrating Geographical and Feminist Analyses of Women's Fear of Crime». Progress in Human Geography, 15(4), 415-431.

Pain, R. (1997). «Social Geographies of Women»s Fear of Crime». Transactions of the Institute of British geographers, 22(2), 231-244.

Pain, R. (2001). «Gender, Race, Age and Fear in the City». Urban studies, 38(5-6), 899-913.

Palmer, B. D. (2000). Cultures of Darkness: Night Travels in the Histories of Transgression (from Medieval to Modern). New York: Monthly Review Press.

Patel, R. (2010). Working the Night Shift: Women in India's Call Center Industry. Stanford University Press.

Rahder, B., \& Altilia, C. (2004). «Where is Feminism in Planning Going? Appropriation or Transformation?». Planning Theory, 3(2), 107-116.

Roberts, M. (2013). «Gender, Fear and the Night-Time City». En De Madariaga, I. S., \& Roberts, M., Fair Shared Cities: The Impact of Gender Planning in Europe. Ashgate Publishing Limited.

Roberts, M. (2006). «Women, Urban Design and Neighborhood Planning». En S. Buckingham \& G. Lievesley, In the Hands of Women. Paradigms of Citizenship. Manchester: Manchester University Press.

— Eldridge, A. (2012). Planning the Night-time City. Routledge.

SAndberG, L., \& Rönnblom, M. (2014). «“I Don't Think We'll Ever Be Finished with this": Fear and Safety in Policy and Practice». Urban Studies, 0042098014550453.

SAndercock, L. (Ed.) (2003). Cosmopolis II: Mongrel Cities of the 21st Century. Bloomsbury Publishing. 
- (2002). «Difference, Fear and Habitus: A Political Economy of Urban Fears». En J. Hillier, \& E. Rooksby (Eds.) (2002), Habitus: A Sense of Place. Aldershot: Ashgate, 203-218.

— (1999). «Expanding the "Language" of Planning: A Meditation on Planning Education for the Twenty-first Century». European Planning Studies, 7(5).

- (Ed.) (1998). Making the Invisible Visible: A Multicultural Planning History. California: University of California Press.

- Forsyth, A. (1992). «A Gender Agenda: New Directions for Planning Theory». Journal of the American Planning Association, 58(1), 49-59.

SChIVELbusch, W. (1988). Disenchanted Night: The Industrialization of Light in the Nineteenth Century. California: University of California Press.

Schwanen, T., van Aalst, I., Brands, J., \& Timan, T. (2012). «Rhythms of the Night: Spatiotemporal Inequalities in the Nighttime Economy». Environment and Planning A, 44(9), 2064.

Sмiтн, D. E. (1990). The Conceptual Practices of Power: A Feminist Sociology of Knowledge. Toronto: University of Toronto Press.

Stanko, E. A. (1988). «Fear of Crime and the Myth of the Safe Home». En K. Yellö \& M. Bograd (Eds.), Feminist Perspectives on Wife Abuse. Sage, Newbury Park, CA.

Stockard, J. (1999). «Gender Socialization». En J. Saltzman Chafetz (Ed.), Handbook of the Sociology of Gender. New York: Kluwer Academic/ Plenum Publishers, pp. 215-227.

Sweet, E. L., \& Ortiz Escalante, S. (2014). «Bringing Bodies into Planning: Visceral Methods, Fear and Gender Violence». Urban Studies, 0042098014541157.

Sweet, E. L., And Ortiz Escalante, S. (2010). «Planning Responds to Gender Violence: Evidence from Spain, Mexico and the United States». Urban studies, 47(10), 2129-2147. 
Tацвот, D. (2007). Regulating the Night: Race, Culture and Exclusion in the Making of the Night-time Economy. Ashgate Publishing, Ltd.

Thomas, C. J., \& Bromley, R. D. (2000). «City-centre Revitalisation: Problems of Fragmentation and Fear in the Evening and Night-time City». Urban Studies, 37(8), 1403-1429.

VALENTiNe, G. (1992). «Images of Danger: Women's Sources of Information about the Spatial Distribution of Male Violence». Area, 24(1), 22-29.

— (1989). «The Geography of Women〉s Fear». Area, 21(4), 385-390.

VARGAS, V. (2009). «La violencia de género: pistas para un análisis». En A. Falú, Mujeres en la Ciudad: De violencias y Derechos. Red Mujer y Hábitat de América Latina. Santiago de Chile: Ediciones Sur.

Waitt, G., Jessop, L., \& Gorman-Murray, A. (2011). «"The Guys in There Just Expect to be Laid": Embodied and Gendered Socio-spatial Practices of a "Night Out" in Wollongong, Australia». Gender, Place and Culture, 18(2), 255-275.

Wesely, J. K., \& GaArder, E. (2004). «The Gendered "Nature" of the Urban Outdoors Women Negotiating Fear of Violence». Gender \& Society, 18(5), 645-663.

Whitzman, C. (Ed.) (2012). Building Inclusive Cities: Women's Safety and the Right to the City. Routledge.

Wicks, P. G., Reason, P. \& Bradbury, H. (2008). «Living Inquiry: Personal, Political and Philosophical Groundings for Action Research Practice». En P. Reason \& H. Bradbury (Eds.), The SAGE Handbook of Action Research. Participative Inquiry and Practice, 199-210.

Wilson, E. (1991). The Sphinx in the City: Urban Life, the Control of Disorder, and Women. Berkeley: University of California Press. 
